\title{
ON THE EXTREMAL ENERGY OF BICYCLIC DIGRAPHS
}

\author{
Mehtab Khan, Rashid Faroog and Azad A. Siddiqui
}

Abstract. The eigenvalues of a graph are the eigenvalues of its adjacency matrix. The energy of a graph is the sum of absolute values of its eigenvalues. Recently, the concept of energy of graphs is extended to digraphs. Minimal and maximal energy among $n$-vertex unicyclic digraphs is known, where $n \geqslant 2$. In this paper, we address the problem of finding minimal and maximal energy among $n$-vertex bicyclic digraphs which contain vertex-disjoint directed cycles, where $n \geqslant 4$.

Mathematics subject classification (2010): 05C35, 05C50.

Keywords and phrases: Energy of digraphs, bicyclic digraphs.

\section{REFERENCES}

[1] G. CAporossi, P. HAnsen, Variable neighborhood search for extremal graphs, 1, The AutoGraphix System, Discrete Math., 212 (2000), 29-49.

[2] D. M. Cvetkovic, M. Doob, H. Sachs, Spectra of Graphs, Academic Press, New York (1980).

[3] I. Gutman, The energy of a graph, Ber. Math. Stat. Sekt. Forschungszentrum Graz, 103 (1978), 1-22.

[4] I. Gutman, Total $\pi$-electron energy of benzenoid hydrocarbon, Topic Curr. Chem., 162 (1992), 2963.

[5] I. Gutman, Acyclic conjugated molecules, trees and their energies, J. Math. Chem., 2 (1987), 123143.

[6] I. Gutman, Acyclic systems with extremal Huckel $\pi$-electron energy, Theor. Chim. Acta, 45 (1977), 79-87.

[7] Y. Hou, Unicyclic grpahs with minimal energy, J. Math. Chem., 29, No. 3 (2001), 163-168.

[8] Y. Hou, I. Gutman, C. W. Woo, Unicyclic grpahs with maximal energy, Linear Alg. Appl., 356 (2002), 27-36.

[9] I. PENA, J. RadA, Energy of Digraphs, Linear Multilinear A., 56, No. 5, (2008) 565-579.

[10] S. PirzadA, M. A. BHAT, Energy of signed digraphs, Discrete Applied Math., 169 (2014) $195-205$.

[11] L. TURKER, An upper bound for total $\pi$-electron energy of alternant hydrocarbons, Commun. Math. Chem., 6 (1984), 83-94. 